\title{
Markov Partition in Non-Hyperbolic Interval Dynamics*
}

\author{
Edson Vargas \\ Departamento de Matemática, PUC/RJ, 22453, Rio de Janeiro, RJ, Brazil \\ Received August 15, 1990; in revised form December 12, 1990
}

\begin{abstract}
We consider $C^{2}$ unimodal maps $f$ such that all periodic points are hyperbolic, the critical point is non-degenerated and non-recurrent, and the Julia set does not contain intervals. We construct a Markov partition for a big part of the Julia set. Then we use it to estimate the limit capacity and Hausdorff dimension of the Julia set.
\end{abstract}

\section{Introduction}

Understanding the dynamical behavior of interval maps is a very interesting task. Besides having amazing and rich dynamics, they are part of more complicated dynamics. We mention some facts about the dynamics of a unimodal map: from the topological point of view we have a good description of the dynamics by the kneading theory, Milnor and Thurston [6]. Melo and Strien [5] and Martens et al. [4] generalized some theorems of structure and finiteness proved before, on the negative schwarzian case, by Guckenheimer [1] and by Singer [7], respectively. From the metrical point of view some problems are still open, for example: the size of invariant compact sets, the relation between stability and hyperbolicity, the existence of Bowen-Ruelle-Sinai measures, etc.

Here we analyze the Julia set $\Sigma$ of unimodal Misiurewicz maps (non-recurrent critical point) with respect to its limit capacity and Hausdorff dimension. Hausdorff dimension is a nice way to measure the size of a set, how much it is dense. Limit capacity relies on geometrical properties of the set, how well it is distributed. Usually limit capacity is bigger than Hausdorff dimension. In the case of $\Sigma$ they are equal. They are bigger than zero and in the case that $\Sigma$ does not contain intervals, they are smaller than one. In fact, when $\Sigma$ does not contain intervals, we can see a lot of order inside it. We can essentially realize it as a compact invariant set of a very nice Markov map.

The paper is divided into three sections. Section 1 contains precise statements and preliminary results. Section 2 is a key one, there we construct the "first hit"

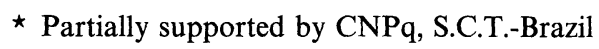


map. It is a Markov map with finite image partition which says almost everything about the size of $\Sigma$ and about the dynamics inside it. In Sect. 3 we use the "first hit" map to estimate limit capacity and Hausdorff dimension.

\section{Statements and Preliminary Results}

Definition. A $C^{r}(r \geqq 2)$ interval map $f:[-1,1] \curvearrowright$ is called unimodal if $f(-1)$ $=f(1)=-1$ and $f$ has only one critical point, zero.

Definition. The basin $B$ of a unimodal map $f$ is the interior of the set of points such that the forward orbit converges to a periodic point. The immediate basin $B_{0}$ is the union of the connected components of $B$ which contain periodic points on its closure. The Julia set $\Sigma$ is the complement of $B$.

Inside the basin the dynamics is very simple, all points are forward asymptotic to a periodic point. The Julia set contains the interesting part of the dynamics, sometimes its dynamics is described by an absolutely continuous invariant measure, Misiurewicz [7].

We consider some unimodal map and assume that its Julia set is small in the topological sense. The following theorem says that it is a small set in a nice metrical and geometrical sense. Let us state the theorem precisely.

Theorem. Let $f$ be a $C^{2}$ unimodal map such that all periodic points are hyperbolic, and its critical point is non-recurrent and non-degenerated. If the Julia set of $f$ does not contain intervals, then:

a) It has Hausdorff dimension bigger than zero and smaller than one.

b) The limit capacity of the Julia set is equal to its Hausdorff dimension.

A key part of the proof of this theorem is the construction of the "first hit" map, in Sect. 2. Besides the metrical information, the "first hit" map explains very well the dynamics inside the Julia set. It will be precisely stated in Sect. 2, as a fundamental lemma.

In a forthcoming paper we consider one-parameter families of unimodal maps through $f$. Although the critical point can be outside the basin, we prove that the frequency of topological changes in the dynamics is zero at the parameter corresponding to $f$.

Now we introduce some notations and some preliminary facts.

Notation. We refer to a $C^{2}$ unimodal map $f$.

a) $B$ denotes the basin, $B_{0}$ the immediate basin, and $\Sigma$ the Julia set of $f$.

b) $x^{*}$ denotes the symmetric point of $x$, that is, the unique point such that $f\left(x^{*}\right)=f(x)$.

c) $J$ denotes an open interval bounded by a point $q$ and its symmetric $q^{*}$. We consider only points $q$ such that $J \cap\left(\bigcup_{j=1}^{\infty} f^{j}(q)\right)=\emptyset$, and for some $m, f^{m}(q)$ is a
periodic point. d) $E_{n}:=\left\{x \in[-1,1] ; f^{j}(x) \notin B_{0} \cup J, j=0, \ldots, n-1\right\}, n \geqq 1$.

Remark. The connected components of $E_{n} \backslash E_{n+1}$ are diffeomorphically applied by $f^{n}$ onto connected components of $B_{0} \cup J$. 
Lemma (1.1). Let $f$ be a $C^{2}$ unimodal map such that all periodic points are hyperbolic. Given $J$ there exist $C_{1}>0, \lambda_{1}>1$ and $R_{1}<\infty$ such that for all $x$ in $E_{n}$ we have:
a) $\left|\partial_{x} f^{n}(x)\right| \geqq C_{1} \lambda_{1}^{n}$
b) $\frac{\left|\partial_{x x} f^{n}(x)\right|}{\left|\partial_{x} f^{n}(x)\right|^{2}} \leqq R_{1}$.

Proof. a) First of all we observe that $E_{n}(n \geqq 1)$ is a decreasing sequence of compact sets, $f^{k}\left(E_{n}\right) \subset E_{n-k}(0<k<n)$ and $\bigcap_{j=1}^{\infty} E_{j}$ is a non-empty compact invariant set of $f$. The set $\bigcap_{j=1}^{\infty} E_{j}$ does not contain attractive periodic points, either non-hyperbolic periodic points or critical points. From Mãné [3] we conclude that $\bigcap_{j=1}^{\infty} E_{j}$ is a hyperbolic set (it also has null Lebesgue measure). Therefore, we can choose some $k$ and some $\bar{\lambda}>1$ such that $\left|\partial_{x} f^{k}(x)\right| \geqq \bar{\lambda}$, for all $x$ in $\bigcap_{j=1}^{\infty} E_{j}$. By continuity we can diminish $\bar{\lambda}$ a little and can take $N$ so big as to have $\left|\partial_{x} f^{k}(x)\right| \geqq \bar{\lambda}$ for all $x$ in $E_{N}=\bigcap_{j=1}^{N} E_{j}$.

Now, given $n>N$ we write $n-N=j k+l$, where $0 \leqq l<k$. Therefore:

$$
\left|\partial_{x} f^{n}(x)\right|=\left|\partial_{x} f^{N+l}\left(f^{j k}(x)\right)\right|\left|\partial_{x} f^{j k}(x)\right| .
$$

For $x$ in $E_{n}$ we have that the first factor is uniformly bounded away from zero and the second is bigger than $(\bar{\lambda})^{j}$. Part a) follows immediately.

b) We observe that:

$$
\frac{\partial_{x x} f^{n}(x)}{\left(\partial_{x} f^{n}(x)\right)^{2}}=\sum_{j=0}^{n-1} \frac{1}{\partial_{x} f^{n-1-j}\left(f^{j+1}(x)\right)} \cdot \frac{\partial_{x x} f\left(f^{j}(x)\right)}{\left(\partial_{x} f\left(f^{j}(x)\right)\right)^{2}} .
$$

The factor $\frac{\partial_{x x} f}{\left(\partial_{x} f\right)^{2}}$ is bounded in the complement of $J$. Now part b) follows by part a).

Lemma (1.2). Let $f$ be a $C^{2}$ unimodal map such that all periodic points are hyperbolic and the critical point (zero) is non-degenerated. Given $J$ there exists $R_{2}<\infty$ such that for all $y$ in $J$ such that $f([0, y]) \subset E_{n-1}$ we have:
a) $\frac{S_{n}}{R_{2}}|y| \leqq\left|\partial_{x} f^{n}(y)\right| \leqq R_{2} S_{n}|y|, S_{n}:=\left|\partial_{x x} f^{n}(0)\right|$.
b) $\frac{S_{n}}{2 R_{2}} y^{2} \leqq\left|f^{n}(y)-f^{n}(0)\right| \leqq \frac{R_{2} S_{n}}{2} y^{2}$.

Proof. We omit this proof which is a straightforward consequence of Lemma (1.1) and the non-degeneracy of the critical point.

\section{The First Hit Map}

This section is a key part of the proof of the theorem. Our first tentative step to prove the theorem is to construct a Markov partition for the Julia set $\Sigma$, but as $\Sigma$ 
may contain the critical point, it is impossible. The following lemma has many dynamical and metrical implications. Roughly speaking it says that we essentially have a Markov partition for $\Sigma$. As a dynamical consequence we can see a Markov chain with infinitely many states inside the dynamics of $f$ in $\Sigma$. As a metrical consequence we can see that $\Sigma$ has null Lebesgue measure. As a geometrical consequence we can see a lot of order inside $\Sigma$ : it is self-similar. In fact, we have much more than this, as we will see in Sect. 3.

Fundamental Lemma (2.1). Let $f$ be a $C^{2}$ unimodal map such that all periodic points are hyperbolic and the critical point is non-degenerated and non-recurrent. If the Julia set $\Sigma$ of $f$ does not contain intervals, there exists a $C^{2} \operatorname{map} \varphi: \bigcup_{j=1}^{k} K_{j} \rightarrow[-1,1]$
( $k$ in $\mathbb{N} \cup\{\infty\}$ ) such that: (a) $K_{j}(j \geqq 1)$ are closed, non-degenerated, disjoint intervals contained in $[-1,1]$. Their boundary points $a_{j}$ and $b_{j}$ form two sequences which converge exponentially to

(b) $\Sigma$ is equal to the union of $\bigcap_{i=0}^{\infty} \varphi^{-i}\left(\bigcup_{j=1}^{k} K_{j}\right)$ and the countable set $\bigcup_{j=0}^{\infty} \varphi^{-j}(0)$.

(c) $\varphi$ is a $C^{2}$ Markov map with finite image partition, that is; $\varphi$ applies each $K_{j} C^{2}$ diffeomorphically onto $\varphi\left(K_{j}\right)$. If $\varphi\left(K_{j}\right)$ intersects $K_{l}$ then $\varphi\left(K_{j}\right)$ contains $K_{l}$. The set of intervals $\left\{\varphi\left(K_{j}\right) ; j \geqq 1\right\}$ is finite.

(d) There exist $C>0, \bar{\lambda}>1$ and $R<\infty$ such that, for all $y$ in the domain of $\varphi^{n}(n \geqq 1)$, we have:

$$
\left|\partial_{x} \varphi^{n}(y)\right| \geqq C \lambda^{n} \quad \text { and } \quad \frac{\left|\partial_{x x} \varphi^{n}(y)\right|}{\left|\partial_{x} \varphi^{n}(y)\right|^{2}} \leqq R .
$$

We refer to the Markov map $\varphi$ as the first hit map. It has nice properties. Before getting into the proof of Lemma (2.1) we say something about the construction of the first hit map. The natural idea is to consider gaps of some fixed piece of the basin. The first hit map could be simply $f$ restricted to these gaps. It works fine, except on the central gap which contains the critical point. To correct it we take its images by iterations of $f$ until hitting the fixed piece of the basin. At this moment we decompose the central gap in some good ones where $\varphi$ is the corresponding iteration of $f$. A new central gap appears. It must go through the correction process. Fortunately this new central gap is considerably smaller, and after infinitely many induction steps all the gaps are nice. We miss only the critical point.

Proof (Lemma (2.1)). We need to build the first hit map $\varphi$ with good topological and metrical properties. We consider three parts; the construction of $\varphi$, its topological properties and its metrical properties.

Part (1)(Construction). Firstly we define the domain $\bigcup_{j=1}^{k} K_{j}$ of $\varphi$ and afterwards we define how $\varphi$ acts on its domain. To define the closed intervals $K_{j}$ we fix a convenient piece of the basin $B$. We have two cases; in the first case $\Sigma$ does not accumulate on the critical point and in the second case $\Sigma$ accumulates on the critical point.

If $\Sigma$ does not accumulate on the critical point (zero), we choose the smallest $j_{0}$ such that the closure of $B_{j_{0}}:=\bigcup_{i=0}^{j_{0}} f^{-i}\left(B_{0}\right)$ contains zero. $B_{j_{0}}$ has finitely many connected components (Martens et al. [4]) and its boundary is invariant by $f$. In 
this easy case the intervals $K_{j}$ are the connected components of the complement of the interior of closure $\left(B_{j_{0}} \cup f^{-1}\left(B_{j_{0}}\right)\right)$. There we take the restriction of $f$ to $K_{j}$ to be the first hit map $\varphi$. Lemma (2.1) follows by Lemma (1.1), by the fact that $B_{j_{0}}$ has finitely many connected components, and by the invariance of the boundary of $B_{j_{0}}$.

Let us assume from now on that $\Sigma$ accumulates on zero. Given some $j_{0}$ we define the interval $\widetilde{J}_{1}$; the open interval bounded by the two symmetric connected components of $B_{j_{0}}$ which are the nearest to zero. We fix $j_{0}$ such that the distance between $\widetilde{J}_{1}$ and $B_{0} \cup\left(\bigcup_{j=1}^{\infty} f^{j}(0)\right)$ is positive. This is possible because $B_{0}$ has finitely many connected components, $\Sigma$ does not contain intervals and zero is nonrecurrent.

Now we start an induction process to define the intervals $K_{j}$ and the first hit $\operatorname{map} \varphi$.

Induction Step (1). $J_{1}:=\widetilde{J}_{1} \backslash B_{j_{0}}$ is a closed non-degenerated interval which contains zero. If we take $k_{0}=0$ we have:

$\left(\mathrm{a}_{1}\right) f^{k_{0}}\left(\partial J_{1}\right) \subset \partial B_{j_{0}}$, here $\partial$ denotes boundary.

$\left(\mathrm{b}_{1}\right) f^{k_{0}}\left(J_{1}\right) \cap B_{j_{0}}=\emptyset$.

Induction Step $(n) . J_{n}$ is a closed non-degenerated interval which contains zero. There exists $k_{n-1}$ such that:

$\left(\mathrm{a}_{n}\right) f^{k_{n-1}}\left(\partial J_{n}\right) \subset \partial B_{j_{0}}$.

(b $f^{k_{n-1}}\left(J_{n}\right) \cap B_{j_{0}}=\emptyset$.

Induction Step $(n+1)$. Because $\Sigma$ does not contain intervals we can choose the smallest $k_{n}$ such that $f^{k_{n}}\left(J_{n}\right) \cap B_{j_{0}} \neq \emptyset$. We define $J_{n+1}$; the maximal interval contained in $J_{n}$ which contains zero and satisfies $f^{k_{n}}\left(J_{n+1}\right) \cap B_{j_{0}}=\emptyset . J_{n+1}$ is closed and non-degenerated and the pair $J_{n+1}$ and $k_{n}$ satisfy $\left(a_{n+1}\right)$ and $\left(b_{n+1}\right)$. This concludes the induction process.

Now we define the domain of the first hit map $\varphi$ and its action on it. The intervals $K_{j}$ are the connected components of $\bigcup_{l=1}^{\infty}\left(J_{l} \backslash\left(J_{l+1} \cup f^{-k_{l}}\left(B_{j_{0}}\right)\right)\right)$ union with $\left([-1,1] \backslash\left(B_{j_{0}} \cup f^{-1}\left(B_{j_{0}}\right)\right)\right) \cap\left([-1,1] \backslash J_{1}\right)$. We enumerate $K_{j}$ conveniently in order that the convex hull of $K_{j}$ and $K_{j+1}$ contains $K_{j+2}$. We define the action of the first hit map $\varphi$ on $K_{j}$; it is $f$ if $K_{j}$ is contained in $[-1,1] \backslash J_{1}$ and $f^{k_{l}}$ if $K_{j}$ is contained in $J_{l} \backslash J_{l+1}$.

Part (2) (Topological Properties). We mean by topological properties those stated in Lemma $(2.1 \mathrm{a}, \mathrm{b}, \mathrm{c})$, except by the exponential convergence of $a_{j}$ and $b_{j}$ to zero; which is a metrical property. All of them are direct consequence of the induction process. We only mention that: $k_{0}<k_{1}<\ldots, \bigcap_{n=1}^{\infty} J_{n}=\{0\}$ and $\varphi$ applies $K_{j}$ diffeomorphically onto $\varphi\left(K_{j}\right)$ which is a connected component of the complement of $B_{j_{0}}$. Since $B_{j_{0}}$ has finitely many connected components and its boundary is invariant by $f$, part (c) in Lemma (2.1) follows.

We have other important topological properties: the fact that each $J_{l} \backslash J_{l+1}$ contains only a finite and uniformly bounded number of intervals $K_{j}$ and the fact that $\left(\bigcup_{l=1}^{k_{n}-1} f^{l}\left(J_{n}\right)\right) \cap\left(B_{j_{0}} \cup J_{1}\right)=\emptyset, n \geqq 1$. 
Part (3) (Metrical Properties). Köbe's lemma (see Strien [9]) is the main tool in this part, at the right moment we point out its role.

We mean by metrical properties the exponential convergence of $a_{j}$ and $b_{j}\left(a_{j}\right.$ and $b_{j}$ are the boundary points of $K_{j}$ ) to zero and part (d) in Lemma (2.1). Part of their proof is the following fact. There exist $\widetilde{C}>0, \tilde{\lambda}>1$ and $\widetilde{R}_{1}, \widetilde{R}_{2}<\infty$ such that for all $y$ in $K_{j} \subset J_{l} \backslash J_{l+1}$ we have $\varphi=f^{k_{l}}$ and:

(i) $\left|\partial_{x} \varphi(y)\right| \geqq \widetilde{C} \tilde{\lambda}^{k_{l}}$ and $\frac{\partial_{x x} \varphi(y) \mid}{\left|\partial_{x} \varphi(y)\right|^{2}} \leqq \tilde{R}_{1}$;

(ii) $\left|J_{l}\right| \leqq \tilde{R}_{2} \tilde{\lambda}^{-k_{l}}$.

To prove (i) we observe that $\varphi=f^{k_{l}}$ in $K_{j} \subset J_{l} \backslash J_{j+1}$ and $f\left(J_{l}\right) \subset E_{k_{l}-1}$. Where $E_{n}:=\left\{x \in[-1,1] ; f^{i}(x) \notin B_{j_{0}} \cup J_{1}, i=0, \ldots, n-1\right\}, n \geqq 1$. By Lemma (1.2), for all $y$ in $K_{j} \subset J_{l}$, we have:

$$
\left|\partial_{x} \varphi(y)\right|=\left|\partial_{x} f^{k_{l}}(y)\right| \geqq \frac{S_{k_{l}}}{R_{2}}|y|
$$

and

$$
y^{2} \geqq \frac{2\left|f^{k_{l}}(y)-f^{k_{l}}(0)\right|}{R_{2} S_{k_{l}}} .
$$

As $y \in J_{l} \backslash J_{l+1}$ the interval bounded by $f^{k_{l}}(y)$ and $f^{k_{l}}(0)$ contains some connected component of $B_{j_{0}}$ and it follows that $\left|f^{k_{l}}(y)-f^{k_{l}}(0)\right|$ is uniformly bounded away from zero. Lemma (1.1) implies that $S_{k_{l}}:=\left|\partial_{x x} f^{k_{l}}(0)\right| \geqq C_{1} S_{1} \lambda_{1}^{k_{l}-1}$. It follows that there exist $\widetilde{C}>0$ and $\tilde{\lambda}>1$ such that $\left|\partial_{x} \varphi(y)\right| \geqq \widetilde{C} \tilde{\lambda}^{k_{l}}$. To prove the boundedness of $\frac{\left|\partial_{x x} \varphi(y)\right|}{\left|\partial_{x} \varphi(y)\right|^{2}}$ we observe that:

$$
\frac{\left|\partial_{x x} \varphi(y)\right|}{\left|\partial_{x} \varphi(y)\right|^{2}} \leqq \frac{1}{\left|\partial_{x} f^{k_{l}-1}(f(y))\right|} \cdot \frac{\left|\partial_{x x} f(y)\right|}{\left|\partial_{x} f(y)\right|^{2}}+\frac{\left|\partial_{x x} f^{k_{l}-1}(f(y))\right|}{\left|\partial_{x} f^{k_{l}-1}(f(y))\right|^{2}}
$$

By Lemma (1.1) the second parcel of this sum is bounded by $R_{1}$. To estimate the first parcel we observe that:

$$
\left|\partial_{x} f^{k_{l}-1}(f(y))\right| \cdot\left|\partial_{x} f(y)\right|^{2}=\left|\partial_{x} \varphi(y)\right|\left|\partial_{x} f(y)\right| .
$$

By an argument analogous to the previous one, we prove that $\left|\partial_{x} \varphi(y) \| \partial_{x} f(y)\right|$ is uniformly bounded away from zero. The proof of (i) is now complete.

By Lemma (1.2) it follows that $y^{2} \leqq \frac{4 R_{2}}{S_{k_{l}}}$. Therefore, we can conclude (ii).

The fact (ii) (above) implies the exponential convergence of $a_{j}$ and $b_{j}$ to zero, but we cannot yet conclude part (d) in Lemma (2.1). We do not have the expansiveness we want; it may fail in some intervals $K_{j}$. By the fact (i) above there exist only finitely many of these $K_{j}$, but this could be enough to cause problems of expansiveness on $\varphi^{n}$. We must correct $\varphi$ where it is not expansive. During this correction process we must not loose the topological and metrical properties already obtained.

Given $\gamma>1$ we redefine $\varphi$ in order to have the following property: If $K_{j}$ is a connected component of the domain of $\varphi$, then:

$$
\varphi\left(K_{j}\right)=J_{1} \quad \text { or } \min \left\{\left|\partial_{x} \varphi(y)\right| ; y \in K_{j}\right\} \geqq \gamma .
$$

We call it property $(\gamma)$. Afterwards we use Köbe's lemma (see Strien [9]) to prove that property $(\gamma)(\gamma>1)$ implies part (d) in Lemma (2.1). 
If $\varphi$ does not have property $(\gamma)(\gamma>1)$ there exist a connected component $K_{j}$ of its domain such that:

$$
\varphi\left(K_{j}\right) \cap J_{1}=\emptyset \text { and } \min \left\{\left|\partial_{x} \varphi(y)\right| ; y \in K_{j}\right\}<\gamma .
$$

We modify $K_{j}$ and redefine $\varphi$ : We choose the smallest $i_{1}$ such that $f^{i_{1}}\left(\varphi\left(K_{j}\right)\right) \cap B_{j_{0}} \neq \emptyset$. The fact (i) above and Lemma (1.1) imply that $\frac{\left|\partial_{x x} f^{i_{1}} \circ \varphi(y)\right|}{\left|\partial_{x} f^{i_{1}} \circ \varphi(y)\right|^{2}} \leqq N$, for all $y$ in $K_{j}$ and some $N<\infty$. In a connected component $\Gamma$ of $K_{j} \backslash\left(f^{i_{1}} \circ \varphi\right)^{-1}\left(B_{j_{0}}\right)$ we redefine $\varphi$ to be $f^{i_{1}} \circ \varphi$. If this new $\varphi$ does not satisfy property $(\gamma)$ with respect to $\Gamma$ we repeat this process: we choose the smallest $i_{2}$ such that $f^{i_{2}}(\varphi(\Gamma)) \cap B_{j_{0}} \neq \emptyset$. As before $\frac{\left|\partial_{x x} f^{i_{2}} \circ \varphi(y)\right|}{\left|\partial_{x} f^{i_{2}} \circ \varphi(y)\right|^{2}} \leqq N$ for all $y$ in $\Gamma$. After finitely many steps we obtain property $(\gamma)$. In fact, in each step the non-linearity of $\varphi$ is bounded uniformly, the size of the image intervals are bounded away from zero and the size of the intervals in the domain becomes very small as the process goes on.

We observe that $\varphi$ must be corrected only in finitely many $K_{j}$ and each one produces finitely many intervals $\Gamma$ where we redefine $\varphi$ and obtain property $(\gamma)$. The complement of the intervals $\Gamma$ in $K_{j}$ is contained in the basin $B$. The topological and metric properties of $\varphi$ are not affected.

Now Köbe's lemma (see Strien [9]) is the key to show that the first hit map $\varphi$, which satisfies property $(\gamma)(\gamma>1)$, has the metrical properties we need in Lemma (2.1). We prove that there exists $n_{0}$ such that $\left|\partial_{x} \varphi^{n_{0}}(y)\right| \geqq \gamma$ for all $y$ in the domain of $\varphi^{n_{0}}$ : we take $y$ in a connected component $K_{j}^{n}$ of the domain of $\varphi^{n}$. We have three cases:

(a) $\varphi^{i}\left(K_{j}^{n}\right) \cap J_{1}=\emptyset, i=0, \ldots, n$. In this case we have $\left|\partial_{x} \varphi^{n}(y)\right| \geqq \gamma^{n}$ by property $(\gamma)$.

(b) $\varphi^{i}\left(K_{j}^{n}\right) \cap J_{1}=\emptyset, i=0, \ldots, n-1$ and $\varphi^{n}\left(K_{j}^{n}\right)=J_{1}$. In this case we take $l$ such that $f^{l}=\varphi^{n}$ in $K_{j}^{n}$. We take also the maximal interval $L_{l}$ which contains $K_{j}^{n}$, where $f^{l}$ is monotone and satisfies $f^{l}\left(L_{l}\right) \cap B_{0}=\emptyset$. By Strien [9], $\sum_{j=0}^{l-1}\left|f^{j}\left(L_{l}\right)\right|$ is uniformly bounded. Since $J_{1}$ is far away from $B_{0} \cup\left(\bigcup_{j=1}^{\infty} f^{j}(0)\right)$ we have that $f^{l}\left(L_{l}\right) \backslash f^{l}\left(K_{j}^{n}\right)$ are two intervals of length bounded away from zero. Then we can use Köbe's lemma (see Strien [9]) to conclude that the non-linearity of $f^{l}=\varphi^{n}$ in $K_{j}^{n}$ is uniformly bounded; that is, $\frac{\left|\partial_{x} \varphi^{n}(y)\right|}{\left|\partial_{x} \varphi^{n}(z)\right|}$ is bounded independently of $n, j$ or $y, z$ in $K_{j}^{n}$. This fact together with the finiteness of image partition of $\varphi$ and the fact that $\left|K_{j}^{n}\right|$ tends uniformly to zero, when $n$ goes to infinity, guarantee the existence of some $n_{1}$ such that $\left|\partial_{x} \varphi^{n_{1}}(y)\right| \geqq \gamma$, for all $y$ in $K_{j}^{n_{1}}$ and any $j$.

(c) $\varphi^{i}\left(K_{j}^{n}\right) \cap J_{1} \neq \emptyset$ for some $i \leqq n-2$. In this case we take the biggest $i \leqq n-2$ such that $\varphi^{i}\left(K_{j}^{n}\right) \cap J_{1} \neq \emptyset$. We have two sub-cases: $i \geqq n_{1}$ or $i<n_{1}, n_{1}$ was fixed in case (b). If $i \geqq n_{1},\left|\partial_{x} \varphi^{n}(y)\right|=\left|\partial_{x} \varphi^{n-i}\left(\varphi^{i}(y)\right)\right|\left|\partial_{x} \varphi^{i}(y)\right| \geqq \gamma^{n-i} \gamma>\gamma$ as we want. If $i<n_{1}$ we have $\left|\partial_{x} \varphi^{i}(y)\right|$ uniformly bounded away from zero and for $n$ big enough we have $\left|\partial_{x} \varphi^{n}(y)\right| \geqq \gamma$ as we want.

Now we can conclude that there exists $n_{0}$ such that $\left|\partial_{x} \varphi^{n_{0}}(y)\right| \geqq \gamma>1$ for all $y$ in the domain of $\varphi^{n_{0}}$. To finish the proof of Lemma (2.1) we show that $\frac{\left|\partial_{x x} \varphi^{n}(y)\right|}{\left|\partial_{x} \varphi^{n}(y)\right|^{2}}$ is uniformly bounded for all $y$ in the domain of $\varphi^{n}$. This follows from the 
boundedness of $\frac{\partial_{x x} \varphi}{\left(\partial_{x} \varphi\right)^{2}}$ proved before in (i), the expansiveness of $\varphi$ and the following formula:

$$
\frac{\partial_{x x} \varphi^{n}(y)}{\left(\partial_{x} \varphi^{n}(y)\right)^{2}}=\sum_{j=0}^{n-1} \frac{1}{\partial_{x} \varphi^{n-1-j}\left(\varphi^{j+1}(y)\right)} \cdot \frac{\partial_{x x} \varphi\left(\varphi^{j}(y)\right)}{\left(\partial_{x} \varphi\left(\varphi^{j}(y)\right)\right)^{2}} .
$$

Corollary (2.2). Let $f$ be as in Lemma (2.1). If the Julia set does not contain intervals it has null Lebesgue measure.

Proof. The proof is a straightforward consequence of Lemma (2.1).

\section{Limit Capacity and Hausdorff Dimension}

In this final section we use the first hit map to estimate the limit capacity and the Hausdorff dimension of the Julia set $\Sigma$. We prove the following theorem that we already stated in the first section.

Theorem. Let $f$ be a $C^{2}$ unimodal map such that all periodic points are hyperbolic and its critical point is non-recurrent and non-degenerated. If the Julia set of $f$ does not contain intervals, then:

(a) It has Hausdorff dimension bigger than zero and smaller than one.

(b) The limit capacity of the Julia set is equal to its Hausdorff dimension.

Besides the dynamical aspects of the first hit map $\varphi$, it implies this fine measurement of the Julia set. The point is that $\varphi^{n}$ produces nice covers of the Julia set. To prove the theorem we need some lemmas.

We consider the first hit map $\varphi$ and the connected components $K_{j}^{n}(j, n \geqq 1)$ of the domain of $\varphi^{n}$. We denote by $k_{n}$ the number of intervals $K_{j}^{n}$. By Lemma (2.1) $k_{n}$ can be all of them finite or all of them infinite. We define a sequence of functions:

$$
H_{n}(t):=\sum_{j=1}^{k_{n}}\left|K_{j}^{n}\right|^{t}, \quad n \geqq 1 .
$$

These functions play a fundamental role in the proof of the theorem. The following lemma says that they have some regularity and decrease at least as an exponential function.

\section{Lemma (3.1).}

(a) $H_{n}(t)$ is defined for all $t$ in $\mathbb{R}^{+}$(i.e. $t>0$ ).

(b) $H_{n}: \mathbb{R}^{+}$๖ is a $C^{1}$ function.

(c) There exist $n_{0}$ and $a>0$ such that $\partial_{t} H_{n}(t) \leqq-a H_{n}(t)$ for all $n \geqq n_{0}$ and $t$ in $\mathbb{R}^{+}$.

Proof. We assume $k_{n}=\infty$, if not; Lemma (3.1) is obvious.

(a) We use induction with respect to $n$. The case $n=1$ is a direct consequence of Lemma (2.1-a). We assume that case $n$ is true and prove that case $n+1$ is also true: for each $K_{l}^{n+1}$ contained in $K_{j}^{1}, \varphi\left(K_{l}^{n+1}\right)$ is some $K_{i}^{n}$ contained in $\varphi\left(K_{j}^{1}\right)$. We define:

$$
\lambda_{n j}:=\min \left\{\left|\partial_{x} \varphi^{n}(y)\right| ; y \text { in } K_{j}^{n}\right\},
$$

for $t>0$ we have:

$$
\sum_{K_{l}^{n+1} \subset K_{J}^{1}}\left|K_{l}^{n+1}\right|^{t} \leqq\left(\frac{1}{\lambda_{1 j}}\right)^{t} \sum_{K_{i}^{n} \subset \varphi\left(K_{j}^{1}\right)}\left|K_{i}^{n}\right|^{t} \leqq \frac{H_{n}(t)}{\left(\lambda_{1 j}\right)^{t}} .
$$


By Lemma (2.1-d) $\frac{1}{\lambda_{1 j}} \leqq \frac{\left|K_{j}^{1}\right|}{\left|\varphi\left(K_{j}^{1}\right)\right|} e^{2 R}$ and, as $\varphi$ has finite image partition, $\left|\varphi\left(K_{j}^{1}\right)\right| \geqq b$; some $b>0$. Therefore, we have:

and part (a) follows.

$$
H_{n+1}(t) \leqq H_{n}(t) H_{1}(t)\left(\frac{e^{2 R}}{b}\right)^{t}
$$

(b) We fix an arbitrary $n$ and consider the following sequence of functions:

$$
h_{l}(t):=\sum_{j=1}^{l}\left|K_{j}^{n}\right|^{t}, \quad t>0 .
$$
When $l$ goes to infinity we have that $h_{l}$ converges to $H_{n}$ uniformly in $\left[\frac{1}{N}, N\right]$,
$N>0$. We claim that the sequence of derivatives;

$$
\partial_{t} h_{l}(t)=\sum_{j=1}^{l}\left|K_{j}^{n}\right|^{t} \log \left|K_{j}^{n}\right|
$$

when $l$ goes to infinity, converges uniformly in $\left[\frac{1}{N}, N\right]$ to:

$$
\sum_{j=1}^{\infty}\left|K_{j}^{n}\right|^{t} \log \left|K_{j}^{n}\right| \text {. }
$$

Let us prove this claim by induction with respect $n$ : For $n=1$ it is a direct consequence of Lemma (2.1-a). If it is true for $n$ we prove it for $n+1$, in fact:

$$
\begin{aligned}
\sum_{k_{l}^{n+1} \subset K_{j}^{1}}\left|K_{l}^{n+1}\right|^{t}|\log | K_{l}^{n+1}|| \leqq & \left(\frac{1}{\lambda_{1 j}}\right)^{t} \sum_{K_{i}^{n} \subset \varphi\left(K_{j}^{1}\right)}\left|K_{i}^{n}\right|^{t}|\log | K_{i}^{n}|| \\
& +\left(\frac{1}{\lambda_{1 j}}\right)^{t} \log \left(\lambda_{1 j} e^{2 R}\right) \sum_{K_{i}^{n} \subset \varphi\left(K_{j}^{1}\right)}\left|K_{i}^{n}\right|^{t} .
\end{aligned}
$$

Now we sum over all $K_{j}^{1}$ and obtain:

$$
\begin{aligned}
\sum_{l=1}^{\infty}\left|K_{l}^{n+1}\right|^{t}|\log | K_{l}^{n+1} \| \leqq & H_{1}(t)\left(\frac{e^{2 R}}{b}\right)^{t} \sum_{i=1}^{\infty}\left|K_{i}^{n}\right|^{t}|\log | K_{i}^{n}|| \\
& +\sum_{j=1}^{\infty}\left(\frac{1}{\lambda_{1 j}}\right)^{t} \log \left(\lambda_{1 j} e^{2 R}\right) \sum_{i=1}^{\infty}\left|K_{i}^{n}\right|^{t}
\end{aligned}
$$

The above sum is uniformly bounded in $\left[\frac{1}{N}, N\right]$. This immediately implies our claim and that $H_{n}$ is differentiable in $\left[\frac{1}{N}, N\right]$. As $N$ is arbitrary we conclude that $H_{n}$ is differentiable in $\mathbb{R}^{+}$. Its derivative is the following continuous function:

$$
\partial_{t} H_{n}(t)=\sum_{j=1}^{\infty}\left|K_{j}^{n}\right|^{t} \log \left|K_{j}^{n}\right|
$$

and part (b) follows. In fact $H_{n}$ is $C^{\infty}$ but we do not need it.

(c) We choose $n_{0}$ big enough to have $\left|K_{j}^{n_{0}}\right| \leqq 1 / 2$, for all $j$. We define:

$$
a:=\min \left\{|| \log \left|K_{j}^{n_{0}} \|\right| j \geqq 1\right\} \text {. }
$$

From the proof of part (b) it follows that $\partial_{t} H_{n}(t) \leqq-a H_{n}(t)$ as we want. 
Corollary (3.2). Let $f$ be as in the theorem. If the Julia set $\Sigma$ does not contain intervals its Hausdorff dimension is smaller than one. Proof. By Lemma (2.1-b) it is enough to prove that $\Lambda:=\bigcap_{n=1}^{\infty}\left(\bigcup_{j=1}^{k_{n}} K_{1}^{n}\right)$ has
Hausdorff dimension smaller than one.

We observe that for each $K_{l}^{m}$ contained in $K_{j}^{n}(m>n \geqq 1), \varphi^{n}\left(K_{l}^{m}\right)$ is some $k_{i}^{m-n}$ contained in $\varphi^{n}\left(K_{j}^{n}\right)$. By an argument similar to the proof of Lemma (3.1-a) we have:

$$
H_{m}(t) \leqq H_{m-n}(t) \cdot H_{n}(t)\left(\frac{e^{2 R}}{b}\right)^{t}
$$

therefore:

$$
H_{l n}(t) \leqq\left(H_{n}(t)\left(\frac{e^{2 R}}{b}\right)^{t}\right)^{l} ; \quad l, n \geqq 1
$$

By Corollary (2.2), $|\Lambda|=|\Sigma|=0$. We know that $K^{n}:=\bigcup_{j=1}^{k_{n}} K_{j}^{n}$ is a decreasing sequence and $\Lambda=\bigcap_{n=1}^{\infty} K^{n}$. Then there exists $n$ such that $H_{n}(1) \cdot \frac{e^{2 R}}{b}<1$. By continuity there exists $\alpha<1$ such that $H_{n}(\alpha) \cdot\left(\frac{e^{2 R}}{b}\right)^{\alpha}<1$. It follows that $H_{l n}(\alpha)$ tends to zero when $l$ goes to infinity. Because $H_{l n}(\alpha)$ is the $\alpha$-Hausdorff measure of the cover $\mathscr{C}_{l n}$ of $\Lambda$, which elements are the intervals $K_{j}^{l n}$, and we know that the Hausdorff dimension of $\Lambda$ is the infimum of all the numbers $d$ such that $\Lambda$ has a cover with arbitrarily small $d$-Hausdorff measure; we can conclude the proof.

Now we prove that the Hausdorff dimension of the Julia set $\Sigma$ is equal to its limit capacity. In fact, we consider only the case that $\Sigma$ accumulates on the critical point zero. If it does not happen, the domain of the first hit map has finitely many connected components and the theorem is a direct consequence of Lemma (2.1) and Takens [10].

Firstly we make an additional assumption: we suppose that $f$ does not have a restrictive central point, in the sense of Guckenheimer [1]. This additional property implies that for each interval $L$ there exists $k_{0}$ such that for all $k \geqq k_{0} f^{k}(L)$ covers all the interval $\left[f^{2}(0), f(0)\right]$; Jonker and Rand [2]. This is a crucial property to get lower bounds to Hausdorf dimension.

Before the following lemma we fix some numbers: Because the first hit map $\varphi$ has finite image partition we can fix some $k_{0}$ such that for all $K_{j}^{n}$ and $k \geqq k_{0}$ we have that $f^{k}\left(\varphi^{n}\left(K_{j}^{n}\right)\right)$ covers $\left[f^{2}(0), f(0)\right]$. We define:

$$
\begin{aligned}
& \lambda_{n j}:=\min \left\{\left|\partial_{x} \varphi^{n}(y)\right| ; y \text { in } K_{j}^{n}\right\}, \\
& \Lambda_{n j}:=\max \left\{\left|\partial_{x} \varphi^{n}(y)\right| ; y \text { in } K_{j}^{n}\right\} .
\end{aligned}
$$

We choose $m_{0}$ such that for all $n \geqq m_{0}, \lambda_{n j} \geqq \tilde{\lambda}>1$. We also define the unique $\Delta_{n}$ and the unique $\delta_{n}$ such that:

$$
\sum_{j \in \mathscr{Z}_{n}}\left(\frac{1}{\Lambda_{n j}}\right)^{\Delta_{n}}=M \text { and } \sum_{j \in \mathscr{L}_{n}}\left(\frac{1}{\lambda_{n j}}\right)^{\delta_{n}}=1,
$$

where $M:=\max \left\{\left|\partial_{x} f^{i}(x)\right| ; i=0, \ldots, k_{0}\right\}$ and

$$
\mathscr{Z}_{n}:=\left\{j \geqq 1 ; K_{j}^{n} \cap\left(f^{2}(0), f(0)\right) \neq \emptyset\right\} .
$$


The following two lemmas are proved only for $n \geqq m_{0}$ because we need that the functions;

$$
\sum_{j \in \mathscr{Q}_{n}}\left(\frac{1}{\Lambda_{n j}}\right)^{t} \text { and } \sum_{j \in \mathscr{D}_{n}}\left(\frac{1}{\lambda_{n j}}\right)^{t}
$$

decrease as $t$ grows. They are essentially the same functions we considered in Lemma (3.1) and for $n \geqq m_{0}$ they decrease at least as an exponential function.

One point about the proof of the two following lemmas is that we can use the first hit map to construct better covers of the Julia set.

Lemma (3.3). Let $f$ be as in the theorem and without restrictive central points. Then the Hausdorff dimension of the intersection of its Julia set with $\left[f^{2}(0), f(0)\right]$ is at least $\Delta_{n}$, for all $n \geqq m_{0}$.

Proof. Let us prove this lemma by contradiction. If the Hausdorff dimension of $\Omega:=\Sigma \cap\left[f^{2}(0), f(0)\right]$ is $\Delta<\Delta_{n}$, there exists $j_{1}$ such that:

$$
\sum_{\substack{j \in \mathscr{P}_{n} \\ j \leqq j_{1}}}\left(\frac{1}{\Lambda_{n j}}\right)^{\frac{\Delta+\Delta_{n}}{2}}>M .
$$

Given $\varepsilon>0, \Omega$ has a cover $\mathscr{C}$ with $\frac{\Delta+\Delta_{n}}{2}$-Hausdorff measure smaller than $M \varepsilon$. We choose $\varepsilon$ small such that each interval in $\mathscr{C}$ intersects at most one $K_{j}^{n}, j \leqq j_{1}$ in $\mathscr{Z}_{n}$. Therefore, $\mathscr{C}$ induces, by restriction, non-empty covers $\mathscr{C}_{j}$ of $\Omega \cap K_{j}^{n}$ and each $\mathscr{C}_{j}$ has strictly less intervals than $\mathscr{C}$.

As the $\frac{\Delta+\Delta_{n}}{2}$ Hausdorff measure of $\mathscr{C}$ is smaller than $M \varepsilon$, there exists some $j \leqq j_{1}$ such that $\frac{\Delta+\Delta_{n}}{\Lambda_{n j}^{2}}$ times the $\frac{\Delta+\Delta_{n}}{2}$-Hausdorff measure of $\mathscr{C}_{j}$ is smaller than $\varepsilon$. Since $f^{k_{0}} \circ \varphi^{n}\left(\Omega \cap K_{j}^{n}\right)$ covers $\Omega$ we can define a new cover $\widetilde{\mathscr{C}}$ of $\Omega$; we take the image of $\mathscr{C}_{j}$ by $f^{k_{0}} \circ \varphi^{n} \cdot \tilde{\mathscr{C}}$ has less intervals than $\mathscr{C}$ and its $\frac{\Delta+\Delta_{n}}{2}$-Hausdorff measure is smaller than $M \varepsilon$.

We can repeat the above process and after a finite number of steps we cover $\Omega$ with an empty cover, which is clearly false.

The point about the proof of the following lemma is that the intervals $K_{j}^{n}$ are nicely distributed and form nice covers of the sets which we are interested in. Given an $\varepsilon>0$ we can count how many intervals of length $\varepsilon$ we need to cover the Julia set or its intersection $\Omega$ with $\left[f^{2}(0), f(0)\right]$. One crucial point is that $K_{j}^{1}$ converges exponentially fast to zero as $j$ goes to infinity. We can get upper bounds to limit capacity. Roughly speaking, the idea is that the intervals $K_{j}^{n}$ provide part of the Julia set with nice covers. Of course they do not have a fixed length as we need in limit capacity but, it does not matter, we can solve this problem: By Lemma (2.1) we know that $K_{j}^{1}$ accumulates at zero. Then given an $\varepsilon>0$ we choose an interval $\mathscr{L}_{0}(\varepsilon)$ of length $\varepsilon$ with center at zero. Outside $\mathscr{L}_{0}(\varepsilon)$ it remains finite many $K_{j}^{1}$, some number of the same order as $|\log \varepsilon|$. We must exchange these $K_{j}^{1}$ by intervals of length $\varepsilon$. If $K_{j}^{1}$ is smaller than $\varepsilon$ we simply replace it by an interval of length $\varepsilon$. If $K_{j}^{1}$ is bigger than $\varepsilon$, we can use the first hit map to reestimate how many intervals of length $\varepsilon$ we need to cover it. At this moment to obtain a better estimate than a 
previous one it is crucial the way that we choose $\delta_{1}$. Unfortunately it is not enough, we need that the function $\sum_{j \in \mathscr{P}_{n}}\left(\frac{1}{\lambda_{1 j}}\right)^{t}$ is strictly decreasing. To be certain about this property we need to consider some high level, $n \geqq m_{0}$ which implies $\lambda_{n j} \geqq \tilde{\lambda}>1$ and the decrease wanted. We need to reestimate more than one time. Let us be more precise from now on.

Lemma (3.4). Let $f$ be as in the theorem and without restrictive central points. Then the limit capacity of the intersection of its Julia set with $\left[f^{2}(0), f(0)\right]$ is at most $\delta_{n}$, for all $n \geqq m_{0}$.

Proof. We prove this lemma by contradiction. If the limit capacity $C(\Omega)$ of $\Omega:=\Sigma \cap\left[f^{2}(0), f(0)\right]$ is $\delta>\delta_{n}$ we first claim that there exist $\varepsilon_{0}>0, \theta_{n}<1$, and $a_{n}<\infty$ such that for all $\varepsilon$ in $\left(0, \varepsilon_{0}\right)$ and $l \geqq 0$ we have:

$$
C(\Omega, \varepsilon) \leqq\left(\left(\sum_{i=0}^{l} \theta_{n}^{i}\right) a_{n} \varepsilon^{\frac{\delta-\delta_{n}}{2}}+\theta_{n}^{l+1}\right) \varepsilon^{-\delta}
$$

where $C(\Omega, \varepsilon)$ denotes the smallest number of intervals of length $\varepsilon$ we need to cover $\Omega$.

Now we assume that the claim is true and prove Lemma (3.4). Afterwards we

prove the claim.
Given $\varepsilon$ in $\left(0, \varepsilon_{0}\right)$ we choose the smallest $l$ such that $\theta_{n}^{l+1}<\varepsilon^{\frac{\delta-\delta_{n}}{2}}$. It follows that:

$$
C(\Omega, \varepsilon) \leqq\left(\left(\sum_{i=0}^{l} \theta_{n}^{i}\right) a_{n}+1\right) \varepsilon^{-\frac{\delta-\delta_{n}}{2}} .
$$

We know that the limit capacity $C(\Omega)$ of $\Omega$ is the infimum of all numbers $d$ for which there exists $\tilde{\varepsilon}>0$, such that, for all $\varepsilon$ in $(0, \tilde{\varepsilon}) \Omega$ has a cover with not more than $\varepsilon^{-d}$ intervals of length $\varepsilon$. The above estimate for $C(\Omega, \varepsilon)$ implies that $C(\Omega) \leqq \frac{\delta+\delta_{n}}{2}<\delta$ which contradicts $C(\Omega)=\delta$.

Now we prove the claim. If $C(\Omega)=\delta$ there exists $\varepsilon_{0}>0$ such that for all $\varepsilon$ in $\left(0, \varepsilon_{0}\right)$ we have that $C(\Omega, \varepsilon) \leqq \varepsilon^{-\delta}$. Given $\varepsilon$ in $\left(0, \varepsilon_{0}\right)$ we use the first hit map to reestimate $C(\Omega, \varepsilon)$. We define:

$$
W_{0}:=\{0\} \text { and } \mathscr{L}_{0}(\varepsilon):=(-\varepsilon / 2, \varepsilon / 2)
$$

and by induction with respect to $n$ :

$$
W_{n+1}:=\varphi^{-(n+1)}(0) \mid \bigcup_{i=0}^{n} \mathscr{L}_{1}(\varepsilon) \text { and } \mathscr{L}_{n+1}(\varepsilon):=\bigcup_{x \in W_{n+1}}(x-\varepsilon / 2, x+\varepsilon / 2) .
$$

We also define:

$$
X_{n}^{\varepsilon}:=\left\{j \in \mathscr{Z}_{n} ; K_{j}^{n} \not \subset \bigcup_{i=0}^{n-1} \mathscr{L}_{i}(\varepsilon) \text { and } \frac{\varepsilon_{0}}{\Lambda_{n j}} \leqq \varepsilon\right\}
$$

and

$$
Y_{n}^{\varepsilon}:=\left\{j \in \mathscr{Z}_{n} ; K_{j}^{n} \not \subset \bigcup_{i=0}^{n-1} \mathscr{L}_{i}(\varepsilon) \text { and } \frac{\varepsilon_{0}}{\Lambda_{n j}}>\varepsilon\right\} .
$$

By Lemma (2.1-a) $K_{j}^{1}$ approximates exponentially fast to zero as $j$ goes to infinity. Therefore, the cardinality of $W_{1}$ is bounded by a constant times $|\log \varepsilon|$. It follows by Lemma $(2.1-\mathrm{a}, \mathrm{d})$ and induction with respect $n$ that the cardinality of $W_{n}$ 
is bounded by a constant times $|\log \varepsilon|^{n}$. Then the number of intervals in $\bigcup_{i=0}^{n-1} \mathscr{L}_{i}(\varepsilon)$ is bounded by a constant times $\sum_{\substack{i=0 \\ n-1}}^{n-1}|\log \varepsilon|^{i}$. The cardinality of $X_{n}^{\varepsilon}$ is bounded by the number of intervals outside $\bigcup_{i=0} \mathscr{L}_{i}(\varepsilon)$, which is at most a constant times $|\log \varepsilon|$. These small $K_{j}^{n}\left(j\right.$ in $X_{n}^{\varepsilon}$ ) can be replaced by intervals of length $\varepsilon$. It remains to cover the part of $\Omega$ inside the big $K_{j}^{n}$, i.e. $j$ in $Y_{n}^{\varepsilon}$. It is in this part that we improve our previous estimate which was $C(\Omega, \varepsilon) \leqq \varepsilon^{-\delta}$, for all $\varepsilon$ in $\left(0, \varepsilon_{0}\right)$. It follows that:

$$
C\left(\Omega \cap \varphi^{n}\left(K_{j}^{n}\right), \varepsilon\right) \leqq \varepsilon^{-\delta}, \quad \varepsilon \text { in }\left(0, \varepsilon_{0}\right) .
$$

Then if we take the pre-image by $\varphi^{n}$ of intervals of length $\varepsilon$ we obtain intervals of length at most $\varepsilon / \lambda_{n j}$. We replace them by intervals of length $\varepsilon / \lambda_{n j}$. It follows that:

$$
C\left(\Omega \cap K_{j}^{n}, \varepsilon\right) \leqq\left(\frac{1}{\lambda_{n j}}\right)^{\delta} \varepsilon^{-\delta}, \quad \varepsilon \text { in }\left(0, \frac{\varepsilon_{0}}{\Lambda_{n j}}\right) .
$$
Then we conclude that to cover the part of $\Omega$ contained in $\bigcup_{j \in \mathcal{Y}_{n}} K_{j}^{n}$ we need not
more than:

$$
\sum_{j \in \mathscr{Z}_{n}}\left(\frac{1}{\lambda_{n j}}\right)^{\delta} \varepsilon^{-\delta}
$$

intervals of length $\varepsilon$. Consequently, we can take $Q<\infty$ such that to cover all the set $\Omega$, with intervals of length $\varepsilon$ in $\left(0, \varepsilon_{0}\right)$, we need not more than

$$
\left(Q \sum_{i=0}^{n}|\log \varepsilon|^{i} \varepsilon^{\delta}+\sum_{j \in \mathscr{P}_{n}}\left(\frac{1}{\lambda_{n j}}\right)^{\delta}\right) \varepsilon^{-\delta}
$$

intervals. We define $a_{n}$ and $\theta_{n}<1$ which we need to prove the claim: $a_{n}$ is the maximum of $\left(Q \sum_{i=0}^{n}|\log \varepsilon|^{i} \frac{\delta+\delta^{2}}{2}\right)$ in $\left(0, \varepsilon_{0}\right)$ and $\Theta_{n}:=\sum_{j \in \mathscr{P}_{n}}\left(\frac{1}{\lambda_{n j}}\right)^{\delta}$. By the way we chose $n$ and $\delta_{n}$ we have that $\theta_{n}<1$. The first reestimate that we have is:

$$
C(\Omega, \varepsilon) \leqq\left(a_{n} \cdot \varepsilon^{\frac{\delta-\delta_{n}}{2}}+\theta_{n}\right) \varepsilon^{-\delta}, \quad \varepsilon \text { in }\left(0, \varepsilon_{0}\right) .
$$

To finish we need some induction. We assume that:

$$
C(\Omega, \varepsilon) \leqq\left(\left(\sum_{i=0}^{l-1} \theta_{n}^{i}\right) a_{n} \frac{\delta-\delta_{n}}{2}+\theta_{n}^{l}\right) \varepsilon^{-\delta} .
$$
As in the first step we can conclude that to cover the part of $\Omega$ in $\underset{j \in Y_{n}}{\bigcup_{n}} K_{j}^{n}$ we need
not more than:

$$
\left(\left(\sum_{i=1}^{l} \theta_{n}^{i}\right) a_{n} \varepsilon^{\frac{\delta-\delta_{n}}{2}}+\theta_{n}^{l+1}\right) \varepsilon^{-\delta}
$$

intervals of length $\varepsilon$. To cover all the set $\Omega$ with intervals of length $\varepsilon$ in $\left(0, \varepsilon_{0}\right)$ we need no more than

$$
\left(\left(\sum_{i=0}^{l} \theta_{n}^{i}\right) a_{n} \varepsilon^{\frac{\delta-\delta_{n}}{2}}+\theta_{n}^{l+1}\right) \varepsilon^{-\delta}
$$

intervals. The claim is proved. 
Corollary (3.5). Let $f$ be as in the theorem and without restrictive central points and let $\Omega$ be the intersection of the Julia set with $\left[f^{2}(0), f(0)\right]$. Then:

(a) The Hausdorff dimension of $\Omega$ is bigger than zero and smaller than one.

(b) The limit capacity of $\Omega$ is equal to its Hausdorff dimension.

Proof. (a) It is the explicit content of Corollary (3.2) and Lemma (3.3).

(b) We know that limit capacity is not smaller than Hausdorff dimension. By Lemma (3.3) and Lemma (3.4) they are between $\Delta_{n}$ and $\delta_{n}, \Delta_{n} \leqq \delta_{n}$ for all $n \geqq m_{0}$. We have:

$$
\sum_{j \in \mathscr{P}_{n}}\left(\frac{1}{\Lambda_{n j}}\right)^{\Delta_{n}}=M \text { and } \sum_{j \in \mathscr{Z}_{n}}\left(\frac{1}{\lambda_{n j}}\right)^{\delta_{n}}=1 .
$$

It follows by Lemma (2.1-d) that:

$$
\sum_{j \in \mathscr{X}_{n}}\left(\frac{1}{\Lambda_{n j}}\right)^{\delta_{n}} \geqq e^{-2 R \delta_{n}}
$$

The $C^{1}$ function $\sum_{j \in \mathscr{Z}_{n}}\left(\frac{1}{\Lambda_{n j}}\right)^{t}\left(n \geqq m_{0}\right)$ decreases at least as an exponential function: in fact, by the same argument as in Lemma (3.1) we have:

$$
\partial_{t}\left(\sum_{j \in \mathscr{Z}_{n}}\left(\frac{1}{\Lambda_{n j}}\right)^{t}\right) \leqq-\left(\log \tilde{\lambda}_{n}\right)\left(\sum_{j \in \mathscr{Z}_{n}}\left(\frac{1}{\Lambda_{n j}}\right)^{t}\right)
$$

where $n \geqq m_{0}$ and $\tilde{\lambda}_{n}:=\min \left\{\lambda_{n j}, j \geqq 1\right\}>1$. It follows that:

$$
\sum_{j \in \mathscr{Z}_{n}}\left(\frac{1}{\Lambda_{n j}}\right)^{t} \leqq M e^{-\left(t-\Delta_{n}\right) \log \tilde{\lambda}_{n}}, \quad t \geqq \Delta_{n} .
$$

Therefore:

$$
\delta_{n}-\Delta_{n} \leqq \frac{2 R \delta_{n}+\log M}{\log \tilde{\lambda}_{n}} .
$$

By Lemma (2.1-d) we know that $\tilde{\lambda}_{n}$ tends to infinity as $n$ goes to infinity, and part (b) follows.

Proof of the Theorem. To start we need some property of the unimodal map $f$. Since its critical point is non-recurrent there exists the minimal restrictive central point $p$. The point $p$ and its symmetric $p^{*}$ define an open interval $J$ and some iterated $f^{n}$ restricted to the closure of $J, C l(J)$, is a unimodal map.

The unimodal map $f^{n}: C l(J) \curvearrowright$ satisfies the hypotheses of Corollary (3.5) and the corresponding set $\Omega$ has the properties obtained there. The Julia set $\tilde{\Sigma}$ of this map is in general an infinite union of compact sets $\widetilde{\Sigma}_{j}(j \geqq 1)$. Each $\widetilde{\Sigma}_{j}$ is applied, by some iterated of $f, C^{2}$-diffeomorphically into $\Omega$. Besides that they approach exponentially fast, as $j$ goes to infinity, to the boundary of $J$. It is because the point $p$ is a hyperbolic periodic one. It follows that $\tilde{\Sigma}$ has the same limit capacity and Hausdorff dimension as $\Omega$.

To finish the proof we observe that the Julia set $\Sigma$ of $f$ is the union of two sets, $\Lambda_{1}$ and $\Lambda_{2}: \Lambda_{1}$ is the set of points in $\Sigma$ for which the forward orbit does not intersect $J$ and $\Lambda_{2}$ is the set of points in $\Sigma$ for which the forward orbit intersects $J$. If we 
consider the map $\Psi$ given by the restriction of $f$ to the complement of $J$, we have that: $\Lambda_{2}=\bigcup_{n=0}^{\infty} \Psi^{-n}(\tilde{\Sigma})$ and $\Lambda_{1}$ is the intersection of the domain of $\Psi^{n}, n \geqq 1$. Now the theorem follows by Lemma (1.1) and Takens [10].

Acknowledgements. I am grateful to Wellington C. de Melo, to Jacob Palis Jr. and Maria E. Vares for many constructive discussions. I am also grateful to the referee for important comments.

\section{References}

1. Guckenheimer, J.: Sensitive dependence on initial conditions for one-dimensional maps. Commun. Math. Phys. 70, 133-160 (1979)

2. Jonker, L., Rand, D.: Bifurcations in one dimension I. Inv. Math. 62, 347-365 (1981)

3. Mãné, R.: Hyperbolicity, sinks and measures in one-dimensional dynamics. Commun. Math. Phys. 100, 495-524 (1985)

4. Martens, M., Melo, W.C., Strien, S.V.: Julia-Fatou-Sullivan theory for real one-dimensional dynamics. Preprint, Delft University (1988)

5. Melo, W.C., Strien, S.V.: A structure theorem in one-dimensional dynamics. Ann. Math. 129, 519-546 (1989)

6. Milnor, J., Thurston, W.: On iterated maps on the intervals I, II. Preprint (1976)

7. Misiurewicz, M.: Absolutely continuous measures for certain maps of an interval. Publ. Math. IHES (1980)

8. Singer, D.: Stable orbits and bifurcations of maps of the interval. SIAM J. Appl. Math. 35, 260-267 (1978)

9. Strien, S.V.: Hyperbolicity and invariant measures for general $C^{2}$ interval maps satisfying the Misiurewicz condition. Commun. Math. Phys. 128, 437-495 (1990)

10. Takens, F.: Limit Capacity and Hausdorff dimension of dynamically defined Cantor sets. Lecture Notes in Mathematics, Vol. 1331, pp. 196-212. Berlin, Heidelberg, New York: Springer 1988

Communicated by Ya. G. Sinai 
